\title{
Effect of Added Mass on the Working Frequency of Slapping Mode Piezoelectric Micro-Jet
}

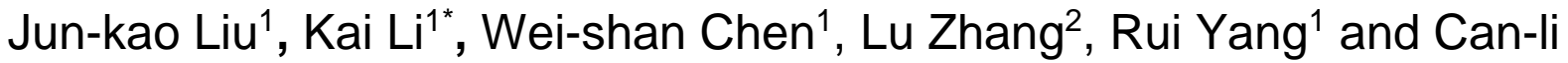 \\ Zhang $^{3}$ \\ 1 Harbin Institute of technology, China \\ 2 AVIC Harin Dong An Engine Corporation \\ 3 Shandong Tai'an Shan-kou Boiler Group Co., Ltd. \\ *sdcxlikai@126.com
}

Keywords: added mass; fluid-structure coupling; working frequency; piezoelectric micro-jet

\begin{abstract}
The piezoelectric micro-jet is a multi-physics coupling device which contains electro-mechanical coupling, fluid-structure coupling and gas-liquid two phase flow coupling. The fluid-structure coupling is mainly referring to the coupling effect between the piezoelectric vibrator and the fluid. Generally, the working frequency of the micro-jet is the resonant frequency of the vibrator which is affected by the fluid-structure coupling. The fluid-structure coupling effect of a kind of micro-jet, which is used for the bearing lubrication, is analyzed in this paper. The added mass is created on the piezoelectric vibrator due to the strong fluid-structure coupling effect, as the vibrator of the micro-jet is immersed in the lubricating oil. The added mass of the piezoelectric vibrator, which directly influences the resonance frequency, is studied in this paper. The relationships between the influence factors and impact strength of the added mass are analyzed and the method of reducing the effect of additional mass is presented. In addition, the adjustment scheme of the working frequency of the micro-jet by taking advantage of the additional mass effect is given.
\end{abstract}

\section{Introduction}

The piezoelectric micro-jet, which can achieve drop-on-demand injection, is widely used in many fields, such as biomedical, electronic circuits, lubrication, 3D printing and chemical [1-5]. The droplets are ejected out of the nozzle under the excitation of pulse voltages, and researchers are focused on the optimization analysis of the excitation according, according to the research on the hydrodynamic characteristics of the micro-jet, to improve the injection performance [6-9]. And, as a sound field is created in the cavity of the micro-jet when the vibrator vibrates, professor D. Bogy proposed to use acoustic theory to analyze the pressure characteristics of the cavity and the nozzle part. [10], thus, refreshers also study on the acoustic characteristics of the micro-jet, in order to optimize the structure dimensions [11].

For the traditional structure form of piezoelectric micro-jet, the piezoelectric vibrator is used as the wall of the cavity, the added mass effect on the vibrator, which is created by the fluid-structure coupling, is weak. But when the vibrator is immersed in the liquid, the added mass effect is enhanced due to the strong fluid structure coupling. In our previous research works, we proposed a kind of piezoelectric micro-jet which works at slapping mode to solving the problem of bearing lubrication failure, and the vibrator of the micro-jet is immersed in the lubricating oil [12]. In order to obtain better injection performance, the piezoelectric vibrator is working in resonant condition. But, the resonance frequency of the vibrator is lower than its natural frequency due to the added mass effect. Then, the working frequency of the micro-jet decreases which result in the reducing of the efficiency.

In order to reduce the added mass effect so as to improve the work efficiency, we analyze the influences of the structure dimensions of vibrator and properties of the lubricating oil on the effect intension of the added mass. And the ways to reduce the effect of the added mass on the working frequency of the piezoelectric micro-jet are given based on the results. Besides, we can adjust the 
working frequency of the micro-jet by utilizing the added mass effect if needed, and the adjustment methods are given as well.

\section{Resonant Frequency of Vibrator with Added Mass}

The relationship between the motion acceleration $\left(a_{0}\right)$ of an object in a vacuum and the external force $(F)$ is described as $a_{0}=F / m$, where $m$ is the mass of the object. When the object is moving in viscous fluid, the motion acceleration $\left(a_{0}^{\prime}\right)$ of this object become less than that in vacuum, and the motion acceleration $\left(a_{0}^{\prime}\right)$ in viscous fluid can be described as $a_{0=}^{\prime} F /\left(m+m^{\prime}\right)$, where $m^{\prime}$ is the added mass of the object when it vibrate in viscous fluid.

As the lubricating oil is a kind of viscous fluid, added mass is created on the vibrator when the vibrator vibrates in the lubricating oil. The diagrammatic sketch of the piezoelectric micro-jet is shown in Fig.1, we can see that, the piezoelectric vibrator can be simplified as a cantilever beam structure with one end fixed and another end immersed in the lubricating oil, and the width of the cantilever is assumed as $b_{0}$.

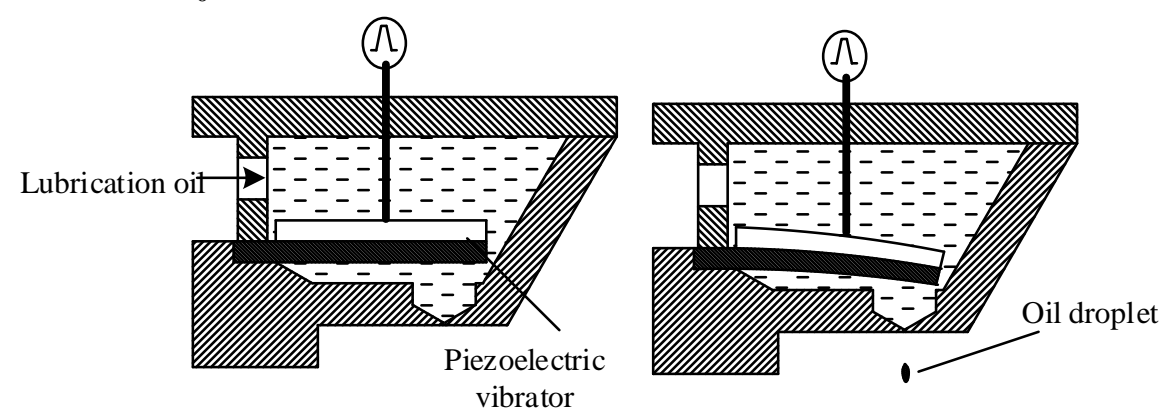

Figure 1. The diagrammatic sketch of the piezoelectric micro-jet

The fluid dynamic equations of a cantilever beam which is immersed in fluid is described as Eq.(1), where $\delta$ represent the thickness of the unstable fluid boundary layer on the surface of the cantilever beam, Re represent the Reynolds number [13]. And $a_{1}=1.0533, a_{2}=3.7997, b_{1}=3.8018, b_{2}=3.7364[14$, $15]$.

$$
\left\{\begin{array}{l}
\Gamma=\Gamma_{r}+j \Gamma_{i} \\
\Gamma_{r}=a_{1}+a_{2} \frac{\delta}{b_{0}}=a_{1}+\frac{a_{2}}{\sqrt{\operatorname{Re}}} \\
\Gamma_{i}=b_{1} \frac{\delta}{b_{0}}+b_{2}\left(\frac{\delta}{b_{0}}\right)^{2}=\frac{b_{1}}{\sqrt{\operatorname{Re}}}+\frac{b_{2}}{\operatorname{Re}}
\end{array}\right.
$$

The thickness of the unstable fluid boundary layer on the surface of the cantilever beam can be calculated by Eq.(2), where $f$ is the vibration frequency of the simplified cantilever beam in lubricating oil, $\rho$ is the density of the lubricating oil and $\eta$ is the dynamic viscosity.

$$
\delta=\sqrt{\frac{2 \eta}{\rho f}}
$$

The Reynolds number can be calculated by Eq.(3), where $f_{0}$ is the resonance frequency of the simplified cantilever beam in vacuum and $b_{0}$ is the width of the simplified cantilever beam.

$$
\operatorname{Re}=\frac{\rho f_{0} b_{0}^{2}}{4 \eta}
$$

The fluid velocity gradient along the radial direction of the piezoelectric vibrator is assumed to be the dominant factor of the fluid velocity, the ratio of the added mass to the mass of the cantilever beam is described as Eq.(4), where $\rho_{\mathrm{b}}$ is the density of the cantilever beam and $h$ is the thickness of the cantilever beam. 


$$
\lambda=\frac{\rho b_{0}}{\rho_{b} h}
$$

Thus, the added mass of the piezoelectric vibrator when it vibrates in the lubricating oil can be calculated by Eq.(5), where $\Delta L$ is the effective length of the vibrator [16].

$$
m_{a}=\rho \frac{\pi}{4} b_{0}^{2} \Delta L \Gamma_{r}
$$

The resonance frequency of the piezoelectric vibrator when it vibrates in the lubricating oil can be calculated by Eq.(6).

$$
f_{w}=\frac{f_{0}}{\sqrt{1+\left(\frac{\pi}{4}\right) \lambda \Gamma_{r}}}
$$

From Eq.(6) we can see that, the resonance frequency of the piezoelectric vibrator with added mass is always less than its natural frequency. And the added mass is related with the density of the lubricating oil and the structure dimensions of the vibrator.

\section{The Influences of Various Factors on Added Mass}

Principle and simplified structure. As we can see form Fig.1, when pulse voltages are applied on the piezoelectric vibrator, the vibrator vibrates in the lubricating oil. And when pressure waves, which are generated by the vibration, are propagated to the nozzle, the lubricating oil droplets are driven out from the nozzle to the bearings. Thus, the piezoelectric vibrator, which determines the operating frequency of the system and is influenced by the added mass, is the key component. In order to find out the relationship between the added mass and the different dimensions of the vibrator by the finite element analysis software ANSYS, we simplify the piezoelectric vibrator as shown in Fig.2.

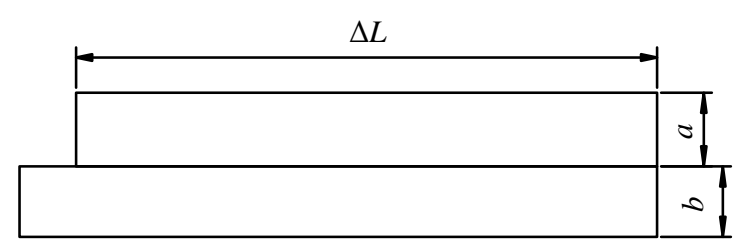

Figure 2. The simplified sturcture of the piezoelectric vibrator

As we can see from Fig.2, the main dimensions of the vibrator include the thickness of the piezoelectric ceramics $(a)$, the thickness of the copper diaphragm $(b)$ and the effective length of the piezoelectric vibrator $(\Delta L)$.

Influences of the thickness of piezoelectric ceramics transducer (PZT). Keep the density of the lubricating oil as $\rho=859 \mathrm{~km} / \mathrm{m}^{3}$, the thickness of the copper diaphragm as $b=0.5 \mathrm{~mm}$ and the effective length of the vibrator as $\Delta L=5 \mathrm{~mm}$, The resonant frequencies of the vibrator, with different thickness of piezoelectric ceramic, in the vacuum and in the lubricating oil are obtained by simulations as shown in Fig.3, where D-value represent frequency of the vibrator vibrates in vacuum minus what is in the lubricating oil. 


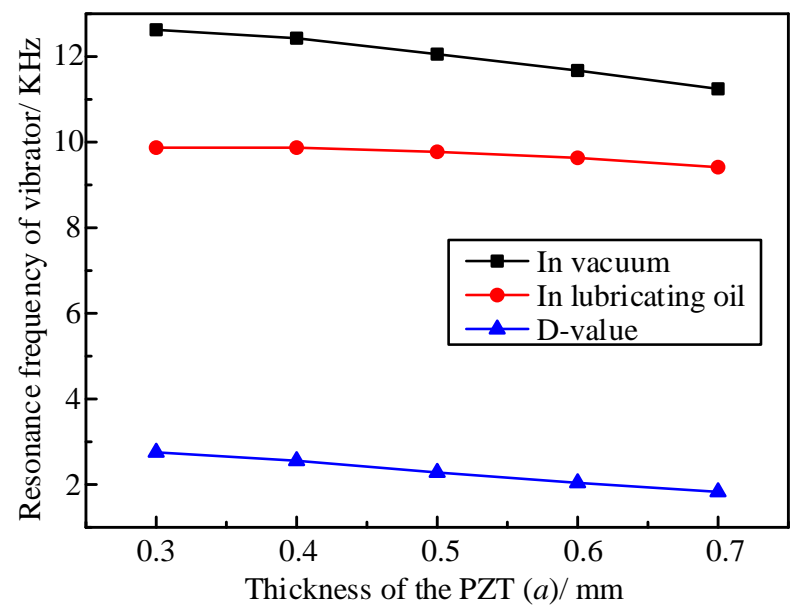

Figure 3. The influences of the thickness of piezoelectric ceramics on the effect intension of the added mass

As we can see form Fig.3, with the increase of the thickness of the PZT, the resonance frequency of the vibrator vibrating in the vacuum decreases gradually, and the resonance frequency of the vibrator, which vibrates in the lubricating oil, is almost keep constant. And, the D-value decreases gradually with the increase of the thickness of the piezoelectric ceramic, thus, the influence of the added mass of the vibrator decreases gradually with the increasing of the thickness of the piezoelectric ceramic.

Influences of the thickness of copper diaphragm. Keep the density of the lubricating oil as $\rho=859$ $\mathrm{km} / \mathrm{m}^{3}$, the thickness of the PZT as $a=0.5 \mathrm{~mm}$ and the effective length of the vibrator as $\Delta L=5 \mathrm{~mm}$, the resonant frequencies of the vibrator varies with different thickness of coper diaphragm are obtained by simulations as shown in Fig.4.

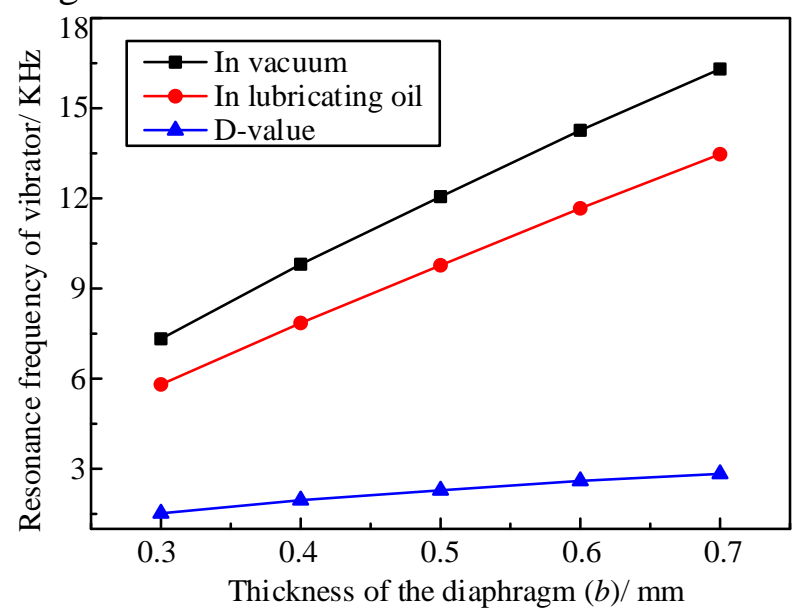

Figure 4. The influences of the thickness of copper diaphragm on the effect intension of the added mass

As we can see form Fig.4, with the increase of the thickness of the copper diaphragm, the resonance frequencies of the vibrator vibrating in the vacuum and the lubricating oil all increase gradually. And, with the increase of the thickness of the copper diaphragm, the D-value increases gradually. Therefore, with the increase of the thickness of the copper diaphragm, the influence of the added mass of the vibrator increases.

Influences of the effective length of the vibrator. Keep the density of the lubricating oil as $\rho=859$ $\mathrm{km} / \mathrm{m}^{3}$, the thickness of the copper diaphragm as $a=0.5 \mathrm{~mm}$ and the thickness of the PZT as $a=0.5 \mathrm{~mm}$, the resonant frequencies of the vibrator varies with different effective length of the vibrator are obtained by simulations as shown in Fig.5. 


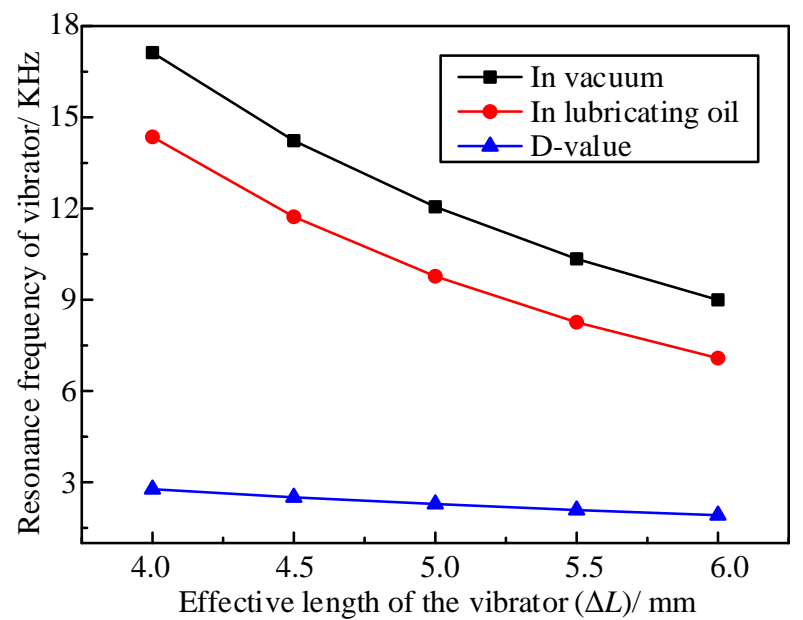

Figure 5. The influences of the thickness of effective lenth of the vibrator on the effect intension of the added mass

As we can see form Fig.5, the resonance frequencies of the vibrator vibrating in the vacuum and the lubricating oil all decrease gradually along with the increase of the effective length of the vibrator. And, with the increase of the effective length of the vibrator, the D-value decrease gradually. Thus, with the increase of the effective length of the vibrator, the influence of the added mass of the vibrator decreases.

Influences of the density of the lubricating oil. As we can see from Eq.(5), the added mass is liner with the density of the lubricating oil. In order to verify the theoretical calculation, the effect of different densities on resonant frequency is analyzed. Keep the thickness of the copper diaphragm and the thickness of the PZT all as $0.5 \mathrm{~mm}$ and the effective length of the vibrator as $\Delta L=5 \mathrm{~mm}$. The resonant frequencies of the vibrator varies with different densities of the lubricating oil are obtained by simulations as shown in Fig.6.

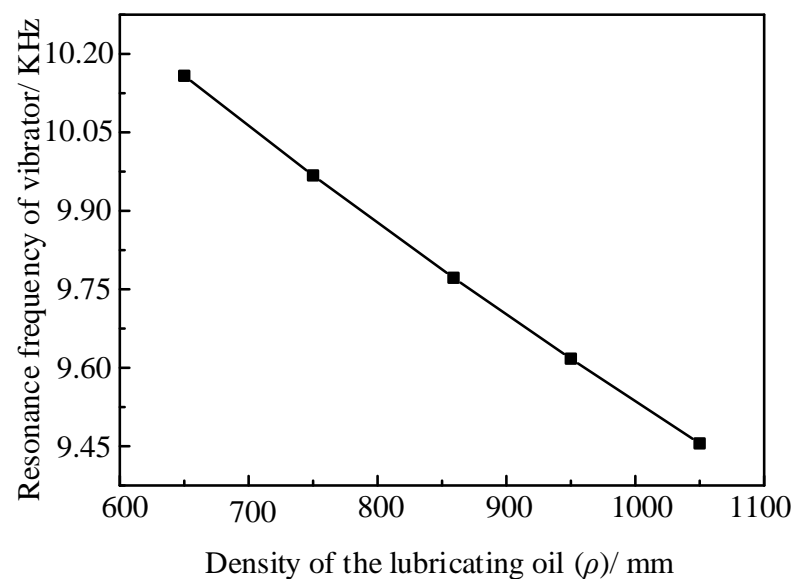

Figure 6. The influences of the density of the lubricating oil on the resonance frequency

As we can see form Fig.6, with the increase of the density of the lubricating oil, the resonant frequency of the vibrator decreases linearly, this is consistent with the theoretical analysis.

\section{Conclusions}

With the permit of the dimensions of the cavity, the thickness of piezoelectric ceramics transducer and the effective length of the vibrator should be raised and the thickness of the copper diaphragm should be decreased in order to reduce the effect of the added mass. If high frequency injection is required to improve the efficiency, the density of the lubricating oil and the effective length of the vibrator should be reduced, and the thickness of copper diaphragm should be increased. The resonance frequency of the vibrator is less affected by the change of ceramic thickness. 


\section{Acknowledgment}

This project was supported by the National Natural Science Foundation of China (No.51075082, No.51375107), all support is gratefully acknowledged.

\section{References}

[1] R. D. Boehm, P. Jaipan, S. A. Skoog, S. Stafslien, L. VanderWal, and R. J. Narayan. 2016. Inkjet deposition of itraconazole onto poly(glycolic acid) microneedle arrays, Biointerphases, 11(1): 011008.

[2] K.-S. Kwon. 2009. Methods for detecting air bubble in piezo inkjet dispensers, Sensors and Actuators A: Physical, 153(1): 50-56.

[3] K. Li, J. Liu, W. Chen, L. Ye, and L. Zhang. 2016. A Novel Bearing Lubricating Device Based on the Piezoelectric Micro-Jet, Applied Sciences, 6(2): 38.

[4] I. Salaoru, Z. X. Zhou, P. Morris, and G. J. Gibbons. 2016. Inkjet printing of polyvinyl alcohol multilayers for additive manufacturing applications, Journal of Applied Polymer Science, 133(25): 9.

[5] K. Kadota, H. Tamura, Y. Shirakawa, Y. Tozuka, A. Shimosaka, and J. Hidaka. 2014. Interfacial sol-gel processing for preparation of porous titania particles using a piezoelectric inkjet nozzle, Chemical Engineering Research \& Design, 92(11): 2461-2469.

[6] K. Li, J. Liu, W. Chen, and L. Zhang. 2016. Optimization of the Excitation signal for a novel piezoelectric micro-jet,

[7] T. M. Liou, C. Y. Chan, and K. C. Shih. 2009. Study of the characteristics of polymer droplet deposition in fabricated rectangular microcavities, Journal of Micromechanics and Microengineering, 19(6): 12.

[8] H. C. Wu, W. S. Hwang, and H. J. Lin. 2004. Development of a three-dimensional simulation system for micro-inkjet and its experimental verification, Materials Science and Engineering a-Structural Materials Properties Microstructure and Processing, 373(1-2): 268-278.

[9] K. Li, J.-k. Liu, W.-s. Chen, L. Ye, and L. Zhang. 2016. Research on the Injection Performance of a Novel Lubricating Device Based on Piezoelectric Micro-Jet Technology, Journal of Electronic Materials, 45(8): 4380-4389.

[10] B. Derby. 2011. Inkjet printing ceramics: From drops to solid, Journal of the European Ceramic Society, 31(14): 2543-2550.

[11] R. Jeurissen, A. van der Bos, H. Reinten, M. van den Berg, H. Wijshoff, J. de Jong, et al. 2009. Acoustic measurement of bubble size in an inkjet printhead, J Acoust Soc Am, 126(5): 2184-90.

[12] K. Li, J.-k. Liu, W.-s. Chen, and L. Zhang. 2016. Effects of pulse voltage on piezoelectric micro-jet for lubrication, Microsystem Technologies, 1-9.

[13] A. Maali, C. Hurth, R. Boisgard, C. Jai, T. Cohen-Bouhacina, and J. P. Aimé. 2005. Hydrodynamics of oscillating atomic force microscopy cantilevers in viscous fluids, Journal of Applied Physics, 97(97): 074907 - 074907-6.

[14] S. B. Basak, S); Raman, A (Raman, A); Garimella, SV (Garimella, SV). 2006. Hydrodynamic loading of microcantilevers vibrating in viscous fluids, JOURNAL OF APPLIED PHYSICS, 99(11): 114906.

[15] A. Ricci, G. Canavese, I. Ferrante, S. L. Marasso, and C. Ricciardi. 2013. A finite element model for the frequency spectrum estimation of a resonating microplate in a microfluidic chamber, Microfluidics and Nanofluidics, 15(2): 275-284.

[16] A. Hossain, A. Mishty, and A. Mian. 2013. Numerical analysis for design optimization of microcantilever beams for measuring rheological properties of viscous fluid, Finite Elements in Analysis and Design, 68(1-9. 